2D pot ent i al measur ements by appl yi ng aut omat i c beam adj ust ment syst em to heavy i on beam probe di agnostic on the Large Hel i cal Devi ce

\begin{tabular}{|c|c|}
\hline $\begin{array}{l}\text { jour nal or } \\
\text { publ i cat } i \text { on } \mathrm{title}\end{array}$ & Revi ew of Sci ent i f i c I nst r ument s \\
\hline vol une & Vol . 85 \\
\hline page $r$ ange & 11D853-1 \\
\hline year & 2014-01- 01 \\
\hline URL & ht t p: //hdl . handl e. net /10655/12480 \\
\hline
\end{tabular}




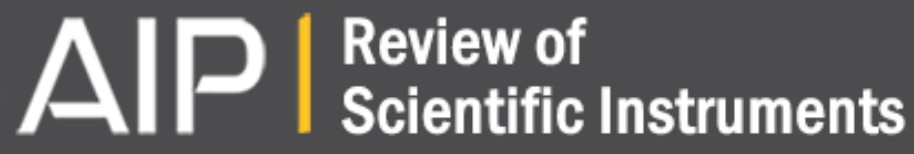

2D potential measurements by applying automatic beam adjustment system to heavy ion beam probe diagnostic on the Large Helical Devicea)

A. Shimizu, T. Ido, M. Kurachi, R. Makino, M. Nishiura, S. Kato, A. Nishizawa, and Y. Hamada

Citation: Review of Scientific Instruments 85, 11 D853 (2014); doi: 10.1063/1.4891975

View online: http://dx.doi.org/10.1063/1.4891975

View Table of Contents: http://scitation.aip.org/content/aip/journal/rsi/85/11?ver=pdfcov

Published by the AIP Publishing

\section{Articles you may be interested in}

Poloidal beam emission spectroscopy system for the measurement of density fluctuations in Large Helical Devicea)

Rev. Sci. Instrum. 81, 10D719 (2010); 10.1063/1.3478685

Magnetic field fluctuation measurement with a heavy ion beam probe in the Large Helical Devicea)

Rev. Sci. Instrum. 79, 10F317 (2008); 10.1063/1.2966376

Reconstruction method of local density fluctuation for heavy ion beam probe measurements

Rev. Sci. Instrum. 78, 063502 (2007); 10.1063/1.2745233

$6 \mathrm{MeV}$ heavy ion beam probe on the Large Helical Device

Rev. Sci. Instrum. 77, 10F523 (2006); 10.1063/1.2338311

Chord integrated neutral particle diagnostic data analysis for neutral beam injection and ion cyclotron radio frequency heated plasma in a complex Large Helical Device geometry

Rev. Sci. Instrum. 77, 10F116 (2006); 10.1063/1.2229279

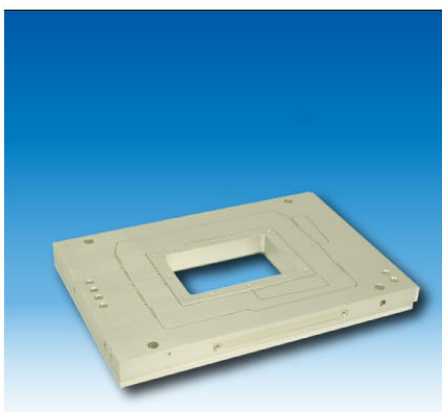

Nanopositioning Systems

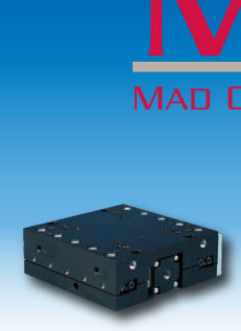

Micropositioning

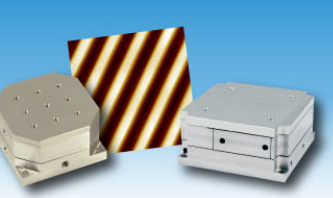

AFM \& SPM

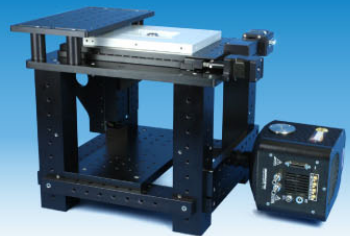

Single molecule imaging 


\title{
2D potential measurements by applying automatic beam adjustment system to heavy ion beam probe diagnostic on the Large Helical Device ${ }^{a)}$
}

\author{
A. Shimizu, ${ }^{1, b)}$ T. Ido, ${ }^{1}$ M. Kurachi, ${ }^{2}$ R. Makino, ${ }^{2}$ M. Nishiura,${ }^{3}$ S. Kato, ${ }^{1}$ A. Nishizawa, ${ }^{4}$ \\ and Y. Hamada ${ }^{1}$ \\ ${ }^{1}$ National Institute for Fusion Science, 322-6 Oroshi, Toki, Gifu 509-5292, Japan \\ ${ }^{2}$ Graduate School of Engineering, Nagoya University, Chikusa, Nagoya 464-8603, Japan \\ ${ }^{3}$ Graduate School of Frontier Sciences, The University of Tokyo, Chiba 277-8561, Japan \\ ${ }^{4}$ Pesco Corporation Limited, Toki, Gifu 509-5123, Japan
}

(Presented 2 June 2014; received 6 June 2014; accepted 21 July 2014; published online 21 August 2014)

Two-dimensional potential profiles in the Large Helical Device (LHD) were measured with heavy ion beam probe (HIBP). To measure the two-dimensional profile, the probe beam energy has to be changed. However, this task is not easy, because the beam transport line of LHD-HIBP system is very long $(\sim 20 \mathrm{~m})$, and the required beam adjustment consumes much time. To reduce the probe beam energy adjustment time, an automatic beam adjustment system has been developed. Using this system, required time to change the probe beam energy is dramatically reduced, such that two-dimensional potential profiles were able to be successfully measured with HIBP by changing the probe beam energy shot to shot. (C) 2014 AIP Publishing LLC. [http://dx.doi.org/10.1063/1.4891975]

\section{INTRODUCTION}

In the confinement physics of magnetically confined toroidal plasmas, the structure of radial electric field $E_{r}$ is important. In this plasma, plasma flow is correlated to $E_{r}$ through $E \times B$ flow. Theoretical research has shown that the shear flow structure can improve the confinement properties by reducing the turbulence in the plasma, ${ }^{1}$ and experimental results that support this theory were obtained in good confinement discharges, such as H-mode, and internal transport barrier (ITB). ${ }^{2,3}$ On the other hand, flow influences appearance and disappearance of islands, thus it is also important for island formation physics. Two-dimensional measurement of $E_{r}$ is helpful for the study of those physics, so the two-dimensional potential measurement with heavy ion beam probe (HIBP) is suggested and reported.

$\mathrm{HIBP}^{4,5}$ is a useful tool for studying the physics related to $E_{r}$, because it can measure the potential in the high temperature plasma with good time and spatial resolution, and without perturbing the plasma. Typically, a one-dimensional profile of potential is measured by varying the incident probe beam angle. By changing the probe beam energy, the observation region is extended and a two-dimensional profile of potential can be measured.

In the Large Helical Device (LHD), an HIBP system was installed and developed to study $E_{r}$ formation physics. ${ }^{6,7}$ This HIBP system has a very long beam transport line $(\sim 20 \mathrm{~m})$, and we have many deflectors and lenses on this line to adjust the beam trajectory along the line. Therefore, it is not easy to change the probe beam energy, because adjustment of the probe beam in the beam transport line is required when the

\footnotetext{
a) Contributed paper, published as part of the Proceedings of the 20th Topical Conference on High-Temperature Plasma Diagnostics, Atlanta, Georgia, USA, June 2014.

b) Author to whom correspondence should be addressed. Electronic mail: akihiro@nifs.ac.jp
}

beam energy is changed, and many deflector voltages must be controlled appropriately. To improve this beam adjustment work and reduce the required time for this adjustment, an automatic adjustment system was developed and applied to our HIBP system. By using this system, the beam trajectory on the beam transport line is automatically adjusted in a short time ( $\sim 3 \mathrm{~min}$, which is a substantial improvement over the several tens of minutes without this system). The probe beam energy is changed shot to shot and a two-dimensional potential profile is measured. In this article, the details of the automatic adjustment system of probe beam in the beam transport line and experimental results of two-dimensional potential measurement are reported.

\section{HEAVY ION BEAM PROBE ON LHD}

The detailed principle of HIBP diagnostics are written in Ref. 5, so only a brief explanation is given here. For an HIBP diagnostic, singly charged particles (called primaries) are injected into the plasma. As the particles pass through the plasma, they are further ionized to produce doubly charged particles (called secondaries). Outside the plasma, the secondaries are detected and their energies are analyzed. Usually the energy analyzer has a slit that allows only secondaries ionized at a given plasma position to reach the detector through the slit. Because of this, measurements localized at the ionization point are possible to obtain. In plasma, primaries of energy $W_{\mathrm{i}}$ are stripped electrons with potential energy of $-e \phi_{\mathrm{sv}}$ by collisions, and change to secondaries that have energy of $W_{\mathrm{i}}+e \phi_{\mathrm{sv}}$. Here, $\phi_{\mathrm{sv}}$ is the electrostatic potential at the sample volume. Therefore, the difference of beam energy between the primaries and the secondaries corresponds to the plasma potential energy $e \phi_{\mathrm{sv}}$ via energy conservation law.

The LHD is a helical device, and its magnetic configuration is characterized by the following parameters, the major radius of the magnetic axis $R_{a x}$, toroidal magnetic field 


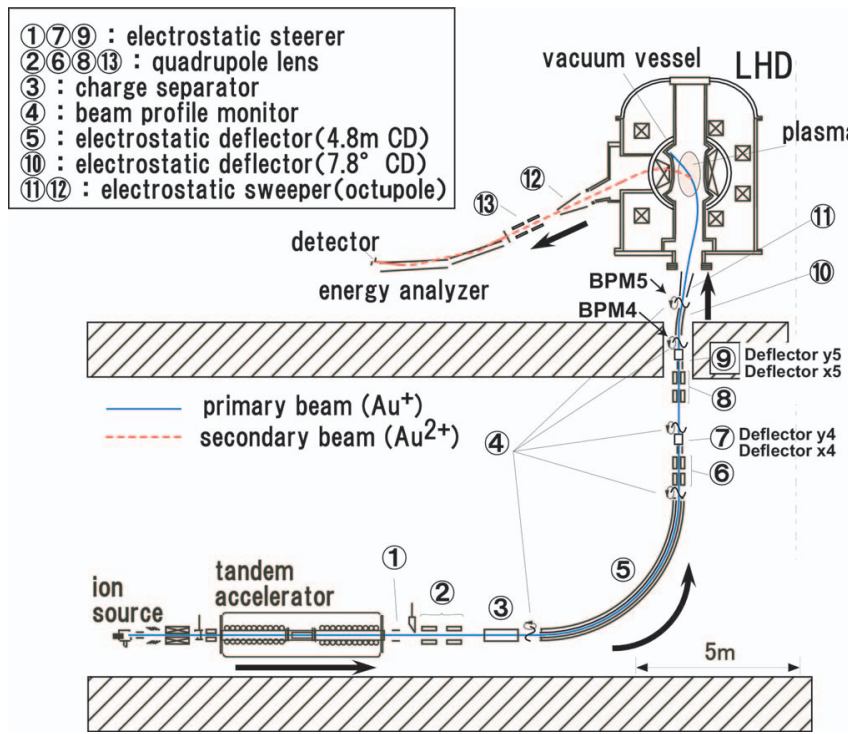

FIG. 1. Schematic view of LHD HIBP system is shown. Notation "CD" in No. 5 and 10 refer to "cylindrical deflector." No. 7 is the deflector $\mathrm{x} 4$ and $\mathrm{y} 4$ and No. 9 is the deflector $x 5$ and $y 5$. BPM 5 is located at the top of the beam transport line, and BPM 4 is the second one from the top.

strength $B_{t}$, the pitch parameter $\gamma$, and the quadrupole component of the magnetic field $B_{q}$. For HIBP diagnostics, the probe beam should be injected to the center of plasma, which requires a high energy probe beam. Because the magnetic field strength of LHD is strong $(\sim 3 \mathrm{~T})$ and the minor radius of plasma is about $0.6 \mathrm{~m}$, we need $\mathrm{MeV}$ energy magnitudes for the probe beam if the gold ion is used. The required energy of the beam reaches $6 \mathrm{MeV}$ for $3 \mathrm{~T}$ of LHD magnetic field strength. To generate such a high energy beam, a tandem accelerator is used in the LHD-HIBP system. By using this accelerator, the voltage required to generate the high energy beam can be decreased by half. To use the tandem accelerator, a negative ion source of gold $\left(\mathrm{A}_{\mathrm{u}}{ }^{-}\right)$is used, and the $\mathrm{A}_{\mathrm{u}}{ }^{-}$ beam is injected into the accelerator. The injected $A_{u}{ }^{-}$beam is accelerated by a voltage of $V_{a c c}$. After that, in the gas cell located at the center of the tandem accelerator tube, the $\mathrm{A}_{\mathrm{u}}{ }^{-}$ ions are stripped of their electrons and changed to $\mathrm{A}_{\mathrm{u}}{ }^{+}$ions. $\mathrm{A}_{\mathrm{u}}+$ ions are re-accelerated by $V_{a c c}$, generating a beam with $2 e V_{a c c}$ energy.

This high energy beam is transported to an injection port of LHD through the beam transport line, of which length is about $20 \mathrm{~m}$. A schematic view of the HIBP system in LHD is shown in Fig. 1. Many electro deflectors and quadrupole lenses are equipped in the beam transport line to adjust the beam orbit. The beam incident angle is varied by the 8-pole electrostatic deflector located at the injection port. At the ejection port, we have another 8-pole electrostatic deflector and adjust the beam direction to a suitable angle to the energy analyzer. In Fig. 2, the observation points with HIBP in the plasma are shown. The actual observation points are arranged three-dimensionally and complicated, so this figure shows a projection of the observation points on the horizontally elongated cross section for simplicity. Usually, the probe beam energy is fixed and the injection/ejection angles are changed to measure a one-dimensional profile (along a line of corresponding beam energy in Fig. 2). If the energy of the probe

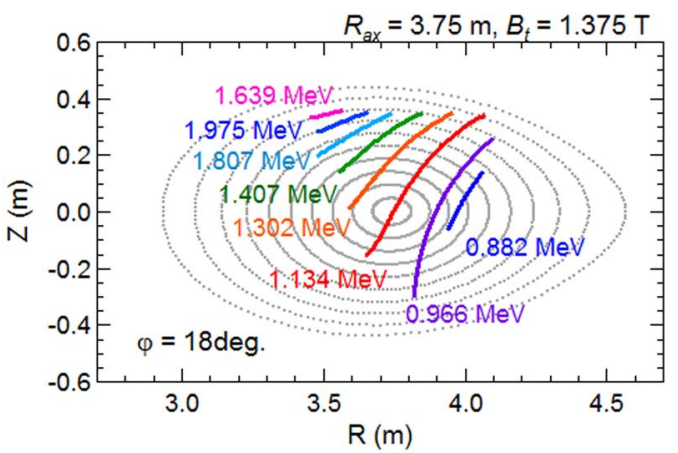

FIG. 2. Observation points with LHD-HIBP system are shown. By changing the injection/ejection angle at fixed probe beam energy, observation points are scanned along a line of corresponding beam energy.

beam is changed, we can measure another one-dimensional profile of the potential. By changing the beam energy shot to shot, two-dimensional potential profile can be obtained.

\section{AUTOMATIC BEAM ADJUSTMENT SYSTEM}

As stated above, the beam transport line of LHD-HIBP system is about $20 \mathrm{~m}$. Therefore, when the probe beam energy is altered, the beam orbit in the beam transport line must also be adjusted. We have many deflectors and lenses in the beam transport line, so many power source voltages for these components are required to be adjusted. These voltages are basically changed in proportion to the probe beam energy, however, effects of magnetic field leakage from the LHD are not negligible so subtle adjustment is needed when the beam energy is changed. To adjust those parameters easily, an automatic adjustment system was developed. In the beam transport line and the accelerator, we have 14 electrostatic deflectors, but it is not easy to include all deflectors into the automatic adjustment system. If many parameters are used, the system consumes large time and tends to be unstable. In the region far from LHD, the effects of magnetic field leakage are sufficiently weak, so 4 deflectors near the injection port are used to optimize the beam orbit, and parameters for other components, such as the quadrupole lens and other deflectors, are changed in proportion to the beam energy. The beam position in the beam transport line is measured by beam profile monitors (BPMs). A schematic view of the BPM detector shown in Fig. 3. The BPM detector comprises a helical

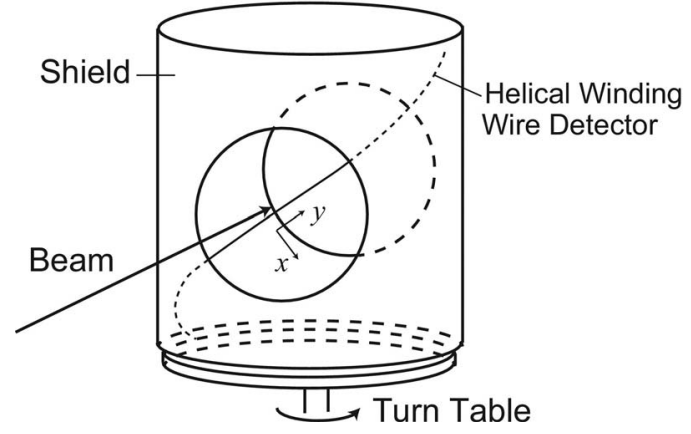

FIG. 3. Detector components of the Beam Profile monitor. The helical winding wire connects to a turntable, which is rotating in measurement process. The wire cuts the beam in $\mathrm{x}$ and $\mathrm{y}$ directions by each rotation of the turntable, so beam profiles in these directions can be measured. 


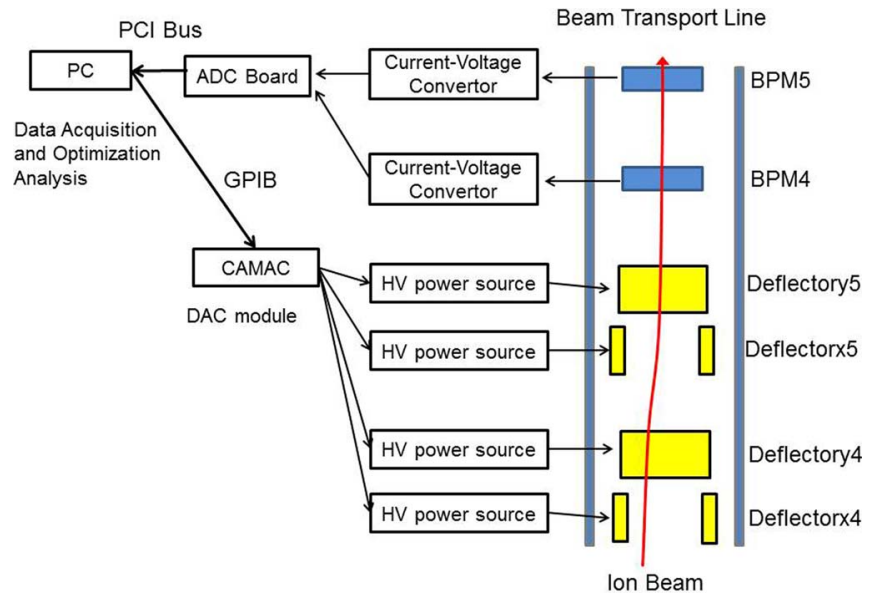

FIG. 4. Simple diagram of PC-based automatic beam adjustment system.

winding wire, a turn table, and a shield. The shield is fixed, and the helical winding wire rotates with the turn table in the measurement process. The beam current is detected with the wire, and beam profiles in two orthogonal axes are measured. Here, displacements of the beam at the point $i$ are defined as $\Delta x_{i}, \Delta y_{i}$ in the $x$ and $y$ axes, respectively. These displacements have basically linear dependence on the deflector voltage $V_{x j}, V_{y j}$. If we use two pairs of $x$ and $y$ deflectors and two BPMs, the displacements of beam can be expressed as

$$
\left(\begin{array}{c}
\Delta x_{4} \\
\Delta y_{4} \\
\Delta x_{5} \\
\Delta y_{5}
\end{array}\right)=\left(\begin{array}{llll}
A_{11} & A_{12} & A_{13} & A_{14} \\
A_{21} & A_{22} & A_{23} & A_{24} \\
A_{31} & A_{32} & A_{33} & A_{34} \\
A_{41} & A_{42} & A_{43} & A_{44}
\end{array}\right)\left(\begin{array}{c}
\Delta V_{x 4} \\
\Delta V_{y 4} \\
\Delta V_{x 5} \\
\Delta V_{y 5}
\end{array}\right) .
$$

Here $\Delta V_{x j}, \Delta V_{y j}$ are the change in the voltage of the deflector $j$. The transport matrix $A_{i j}$ can be calculated theoretically, but in the actual optimization, components of the transport matrix $A_{i j}$ obtained from calibration experiments were used.

A simple diagram of the PC-based optimization system is shown in Fig. 4. The ADC board, which operates at a sample frequency of $1 \mathrm{kHz}$, is installed into a PCI slot in the PC. The signal from the BPM is acquired through this $\mathrm{ADC}$, and the displacements $\Delta x_{i}, \Delta y_{i}$ are calculated. The required deflector voltage to shift the beam position to the center of beam transport line can be calculated using the inverse matrix of $A_{i j}$, as $\mathbf{\Delta} \mathbf{V}=\mathbf{A}^{-1} \boldsymbol{\Delta} \mathbf{x}$. The calculated voltages for deflector high voltage power sources are applied through CAMAC DAC modules connected through general purpose interface bus (GPIB) controller with the PC. After that, the probe beam position is measured with the BPM again, and the next optimization loop is repeated. When distances from the beam transport line axis at the BPMs become less than $1 \mathrm{~mm}$, the optimization process stops. Visual Basic is used to develop our system on the PC. Using the automatic beam adjustment system, the required time to optimize the beam in the beam transport line can be reduced, and we can finish this optimization work within $3 \mathrm{~min}$ (without this system, we needed several tens of minutes for the manual optimization process). In LHD, discharges are repeated every $3 \mathrm{~min}$, so we can change the beam probe energy between each discharge.

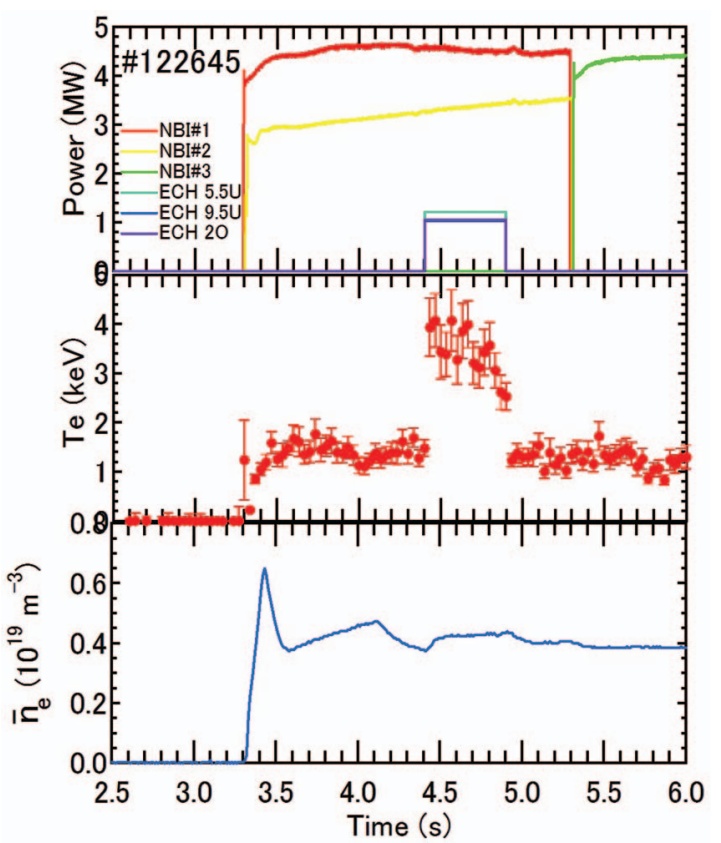

FIG. 5. Temporal evolutions of typical plasma parameters in which twodimensional potential profiles are measured.

\section{EXPERIMENTAL RESULTS}

Using the automatic beam adjustment system, the beam probe energy is changed shot to shot, and the two-dimensional potential profile is measured. Parameters of the magnetic configuration of LHD are as follows: $B_{t}=1.375 \mathrm{~T}, R_{a x}=3.75 \mathrm{~m}$, and $B_{q}=100 \%$. Time evolutions of typical plasma parameters are shown in Fig. 5. The plasma is produced and sustained by neutral beam injection (NBI) heating. Auxiliary heating of electron cyclotron heating $(\mathrm{ECH})$ is applied from 4.4 to $4.9 \mathrm{~s}$. The central electron temperature is about $1.5 \mathrm{keV}$ in the NBI phase, and it is $4 \mathrm{keV}$ in the $\mathrm{NBI}+\mathrm{ECH}$ phase. The line average electron density is about $0.4 \times 10^{19} \mathrm{~m}^{-3}$. In seven similar shots, the probe beam energy is varied shot to shot and the two-dimensional potential profile is measured in a NBI+ECH phase (4.6-4.7 s). In Fig. 6, the obtained potential profile is shown with magnetic surfaces. As the actual HIBP observation points are arranged in three-dimensional space, we plot their projection on the horizontally elongated cross section in this figure for simplicity. The two-dimensional equilibrium potential profile is successfully obtained. In Fig. 7, potential

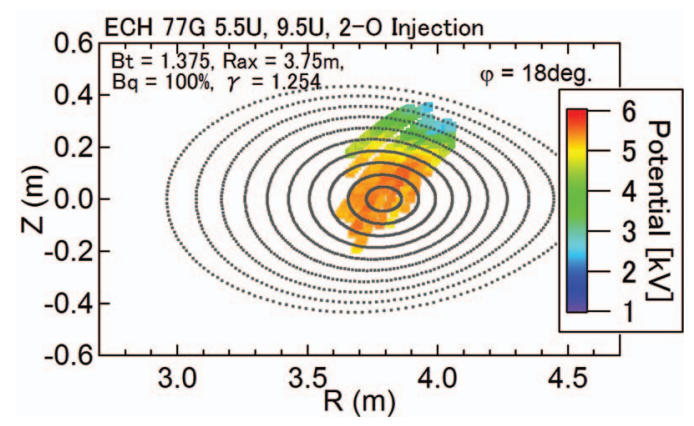

FIG. 6. Two-dimensional potential profile in the ECH applied phase measured with HIBP is shown. 


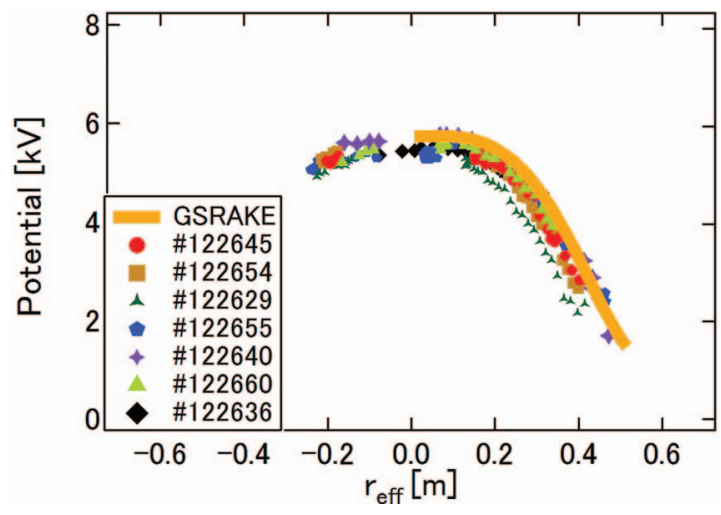

FIG. 7. Potential profiles as a function of averaged normalized minor radius are shown. The 2D profile in Fig. 6 is obtained from these seven shots.

profiles as a function of effective minor radius, $r_{\text {eff }}$, in these seven shots are shown. The potential profile in the inner region is flat, which coincides with the electron temperature profile measured with Thomson scattering diagnostics.

The measured potential profiles are compared with the neoclassical prediction calculated by GSRAKE code. ${ }^{8}$ Experimental results coincide well with the neoclassical prediction, and this tendency is consistent with previous experimental results. ${ }^{9}$

\section{SUMMARY}

In the LHD-HIBP system, an automatic beam adjustment system to easily optimize the beam orbit in the beam transport line has been developed. By using this system, we can change the probe beam energy within $3 \mathrm{~min}$, which corresponds to the interval of LHD discharges, while it takes several tens of minutes without this system. By applying the system to experiments, the probe beam energy is changed shot to shot and two-dimensional potential profiles are successfully measured.

\section{ACKNOWLEDGMENTS}

This work was supported by the NIFS under Contract Nos. ULHH 020 and 023, and by JSPS KAKENHI Grant Nos. 24561031 and 25820441. The authors are grateful for the continuous support of the scientific and technical staff in the LHD experiment group.

${ }^{1}$ K. H. Burrell, Phys. Plasmas 4, 1499 (1997).

${ }^{2}$ A. Fujisawa, H. Iguchi, T. Minami et al., Phys. Rev. Lett. 82, 2669 (1999).

${ }^{3}$ T. Ido, K. Kamiya, Y. Miura, Y. Hamada, A. Nishizawa, and Y. Kawasumi, Phys. Rev. Lett. 88, 055006 (2002).

${ }^{4}$ F. C. Jobes, J. F. Marshall, and R. L. Hickok, Phys. Rev. Lett. 22, 1042 (1969).

${ }^{5}$ T. P. Crowley et al., IEEE Trans. Plasma Sci. 22, 291 (1994).

${ }^{6}$ T. Ido, A. Shimizu, M. Nishiura, A. Nishizawa, S. Katoh, K. Tsukada, M. Yokota, H. Ogawa, T. Inoue, Y. Hamada et al., Rev. Sci. Instrum. 77, 10F523 (2006).

${ }^{7}$ A. Shimizu, T. Ido, M. Nishiura, H. Nakano, I. Yamada, K. Narihara, T. Akiyama, T. Tokuzawa, K. Tanaka et al., J. Plasma Fusion Res. 2, S1098 (2007).

${ }^{8}$ C. D. Beidler and W. D. Dhaseller, Plasma Phys. Controlled Fusion 37, 463 (1995).

${ }^{9}$ A. Shimizu, T. Ido, M. Nishiura, S. Nakamura, M. Nakamura, H. Nakano, M. Yokoyama et al., in Proceedings of the 23rd IAEA Fusion Energy Conference, Daejeon, 11-16 October 2010, EXC/P4-11. 